\title{
An expanding Universe
}

\author{
Astronomers, astrophysicists and planetary scientists are global citizens who transcend political \\ boundaries. Nature Astronomy supports a strong, open community with common interests.
}

Welcome to the first issue of

Nature Astronomy, a new journal for astronomers, astrophysicists and planetary scientists. Reflecting an ever shifting and expanding research field, the publishing landscape for astronomy is also changing. Collaborations are growing bigger and traditionally indifferent communities are coming together, not building walls. Take exoplanets, for example. The search for planets beyond our Solar System was originally the domain of astronomers. But as explained by Andrew Ingersoll in a Review (article no. 0010) the exoplanetary exploration effort can learn from the planetary exploration community, and vice versa.

By covering the full breadth of astronomy, including its societal impact, we hope that readers will come to find in our journal major advances in research in their own subject areas as well as progress in related areas that are interesting and significant. In this way, different communities may discover similarities and shared interests. The Comment by Abraham Loeb and Nia Imara (article no. 0006) explores the idea that collaborations between scientists working at different length scales are more likely to find underlying fundamental principles.

As Nature Research, we publish a series of distinct but related journals. Nature has covered astronomical discoveries since its launch in 1869 (see our web collection of some of the 'greatest hits' in astronomy: www.nature.com/astrohighlights), and that is not about to change. Also, our sister titles Nature Physics and Nature Geoscience will continue to feature astrophysics and planetary science, respectively, for the communities that they serve. With the addition of Nature Astronomy, we take nothing away - rather, we offer more authors the opportunity to publish their papers to a wide audience of astronomers, alongside expert comment and review in the many regular sections of a Nature journal, including News \& Views, Perspective and Review. And in a new section for Nature Astronomy, Mission Control, we will be putting the spotlight on a different observatory each month.

So why choose Nature Astronomy? We provide a curated monthly issue, online only, that is dedicated to the beauty and mystery of 'out there'. For authors, our editorial process is free, fair and fast: we aim to provide initial decisions within one week, we do not have publication charges and we select 2-3 referees per paper. Our editorial team comprises four full-time editors with expertise ranging from our planetary system to the outer reaches of the Universe. We edit the papers to be as accessible to the widest audience possible within our community and we help them reach a wide audience through our press office and our sister titles.

And we certainly intend to promote all the glorious images that astronomers produce. We can even publish videos as standalone pieces in our Video section, with their own digital object identifiers (and not hidden away as Supplementary Information). Please watch our video on Ceres (article no. 0030) as a first example of many to come. It describes the discovery of water ice in the permanent shadows of the northern polar region (article no. 0007), thus establishing the presence of a shadowy cold trap in analogy with those on the Moon and Mercury.

In terms of spreading the word, we appreciate the role of preprint servers in the astronomy community: authors are welcome to post the original version of their manuscript to arXiv at any time. And following publication, authors may also claim a SharedIt link to their paper, which they are welcome to distribute among colleagues for free access to a readable PDF version of the work (please see http://www.springernature.com/ sharedit for more information on our free content sharing initiative). Our inaugural issue will itself be free to access online for the next year.

As we aim to foster a strong community spirit, we welcome feedback and suggestions. We also sponsor conferences and poster prizes, so please get in touch. There are many ways to reach us: follow us on Twitter (@NatureAstronomy), send an e-mail (natureastro@nature.com) or come and say hello when you see one of us (pictured below) at a conference.
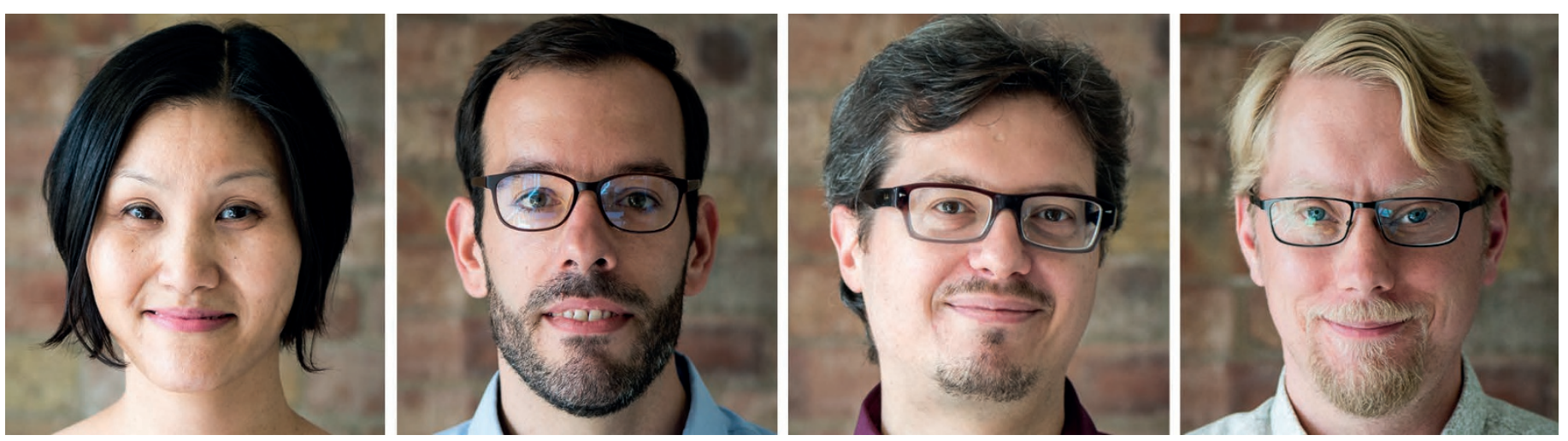

From left to right: May Chiao, Marios Karouzos, Luca Maltagliati and Paul Woods. 